\title{
Uma causa incomum de dor posterior no joelho: Degeneração mucóide do ligamento cruzado anterior - Relato de caso*
}

\section{An Unusual Cause of Posterior Knee Pain: Mucoid Degeneration of the Anterior Cruciate Ligament - Case Report}

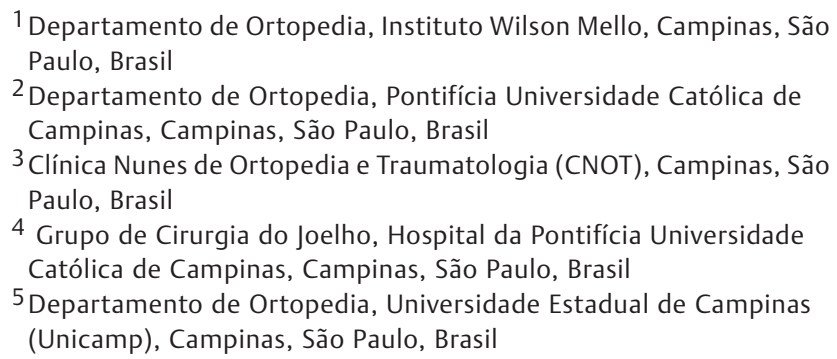

Fabiano da Silva Marques ${ }^{1,4(0)}$ Pedro Bezzan Rodrigues Alves ${ }^{1,4(0)}$ Sandro Zelada ${ }^{1,4}$

Rodrigo Pereira da Silva Nunes ${ }^{2,3(0)}$ Wilson Mello Alves ${ }^{1,4(0)}$ Gustavo Constantino de Campos ${ }^{1,5(0)}$

Endereço para correspondência Fabiano da Silva Marques, MD, Avenida Nelsia Vannucci, 105, apto. 33, Campinas, São Paulo, 13042104, Brasil (e-mail: fabiano.s.marques@gmail.com).

Rev Bras Ortop

\section{Resumo \\ Palavras-chave \\ - ligamento cruzado anterior \\ - artroscopia \\ - articulação do joelho}

A degeneração mucoide do ligamento cruzado anterior (LCA) é uma causa pouco frequente de dor na região posterior do joelho, de patofisiologia desconhecida e subdiagnosticada. A melhor modalidade de tratamento ainda está em discussão. A ressecção da lesão com desbridamento parcial do LCA tem apresentado bons resultados, sem a ocorrência de instabilidade. Os autores apresentam um caso de degeneração mucoide do LCA tratado com ressecção da degeneração mucoide e desbridamento parcial do LCA por artroscopia.

Mucoid degeneration of the anterior cruciate ligament (ACL) is an uncommon cause of pain in the posterior region of the knee, of unknown pathophysiology and underdiagnosed. The best treatment modality is still under discussion. Resection of the lesion with partial $\mathrm{ACL}$ debridement has shown good results, without the occurrence of instability. The authors present a case of mucoid degeneration of the ACL treated with resection of the mucoid degeneration and partial debridement of the ACL by arthroscopy.
Trabalho desenvolvido nos Departamentos de Ortopedia da Universidade Estadual de Campinas (Unicamp) e do Instituto Wilson Mello, Campinas, São Paulo, Brasil. recebido

18 de Julho de 2020

aceito

16 de Setembro de 2020
DOI https://doi.org/

10.1055/s-0040-1722593. ISSN 0102-3616. (c) 2021. Sociedade Brasileira de Ortopedia e Traumatologia. All rights reserved.

This is an open access article published by Thieme under the terms of the Creative Commons Attribution-NonDerivative-NonCommercial-License, permitting copying and reproduction so long as the original work is given appropriate credit. Contents may not be used for commercial purposes, or adapted, remixed, transformed or built upon. (https://creativecommons.org/ licenses/by-nc-nd/4.0/)

Thieme Revinter Publicações Ltda., Rua do Matoso 170, Rio de Janeiro, RJ, CEP 20270-135, Brazil 


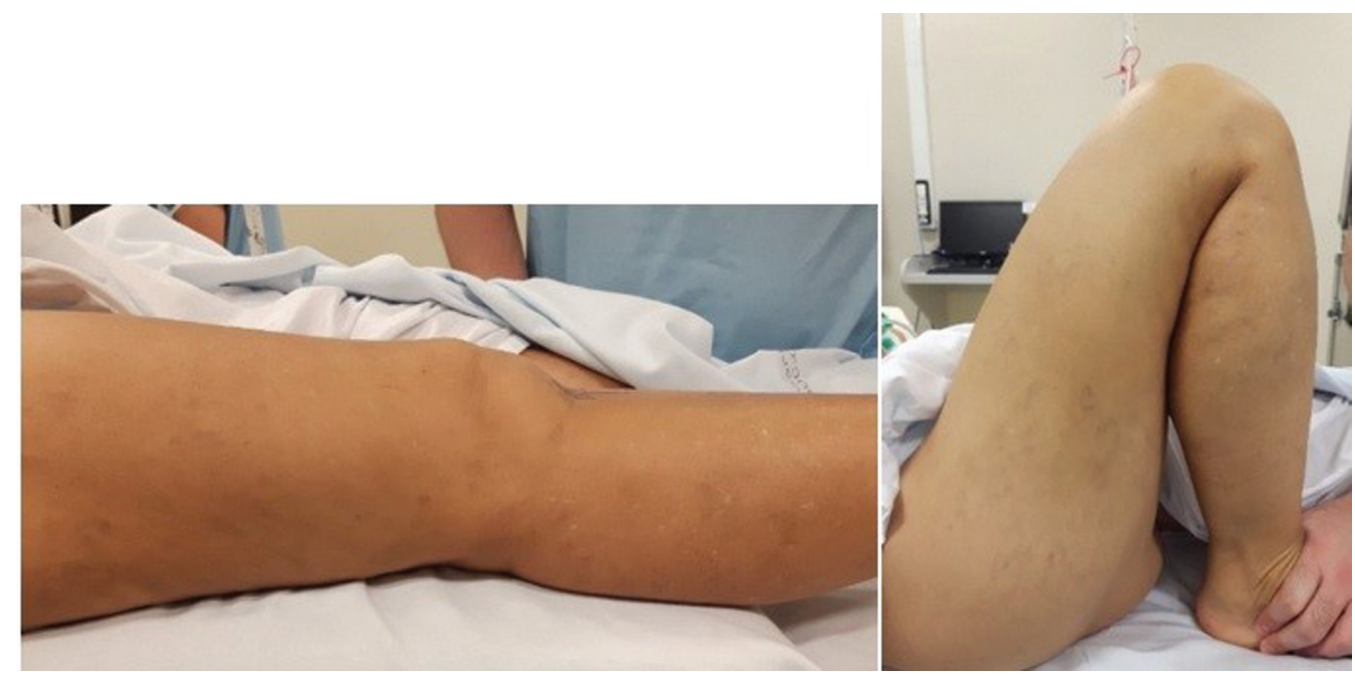

Fig. 1 Imagem clínica pré-operatária.

\section{Introdução}

A degeneração mucoide do ligamento cruzado anterior (LCA) é uma entidade rara, de patofisiologia desconhecida, e subdiagnosticada e subtratada em nosso meio. ${ }^{1,2} \mathrm{O}$ primeiro caso foi descrito por Kumar et al. ${ }^{3}$ Desde então, múltiplos artigos vêm abordando e dando relevância ao tema. ${ }^{1-10}$

\section{Relato de Caso}

Paciente do sexo feminino, de 51 anos, queixando-se de dor em região posterior de joelho direito havia um ano. Ela negava traumas prévios, e seus antecedentes pessoais e familiares eram irrelevantes.
Ao exame físico, a paciente apresentava ausência de derrame articular ou edema, arco de movimento de $0^{\circ}$ à $130^{\circ}$ ( - Figura 1), e dor em região posterior de joelho direito à flexão máxima. Observou-se ausência de sinais e testes clínicos sugestivos de instabilidade ou de lesões meniscais, e as radiografias de joelho de rotina não apresentavam alterações relevantes (-Figura $\mathbf{2}$ ).

Fisioterapia motora para alongamento e fortalecimento do quadríceps associada a anti-inflamatório não esteroidal (AINE) foi prescrita, sem benefício. A ressonância magnética (RM) (-Figura 3) evidenciou espessamento e alteração de sinal do LCA em toda a sua extensão, mantendo a topografia e o paralelismo de suas fibras. Oberservou-se também alteração de sinal no corpo e corno posterior do menisco medial.
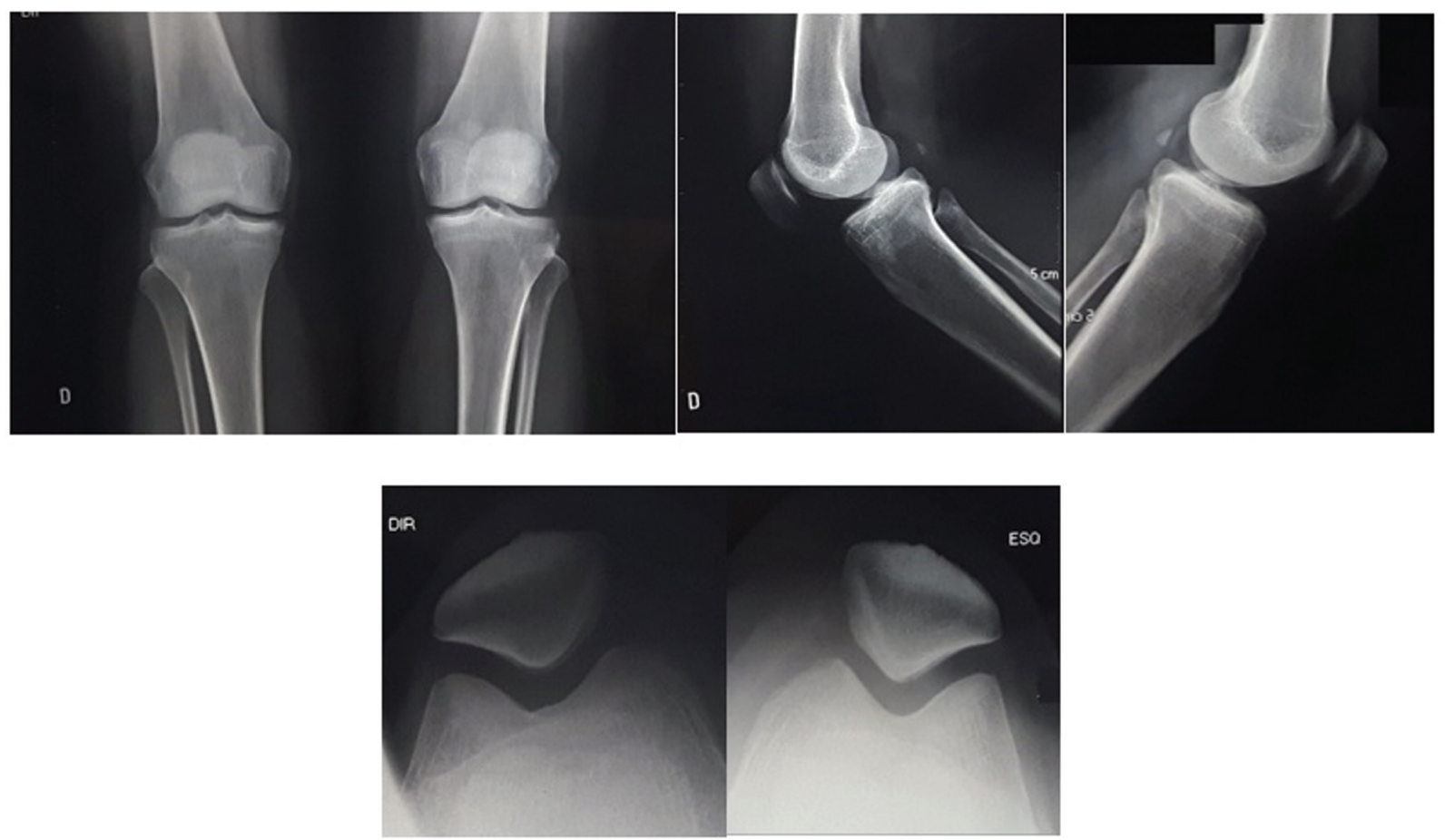

Fig. 2 Radiografias pré-operatórias em nas incidências anteroporsterior, perfil, e axial da patela de ambos joelhos. 


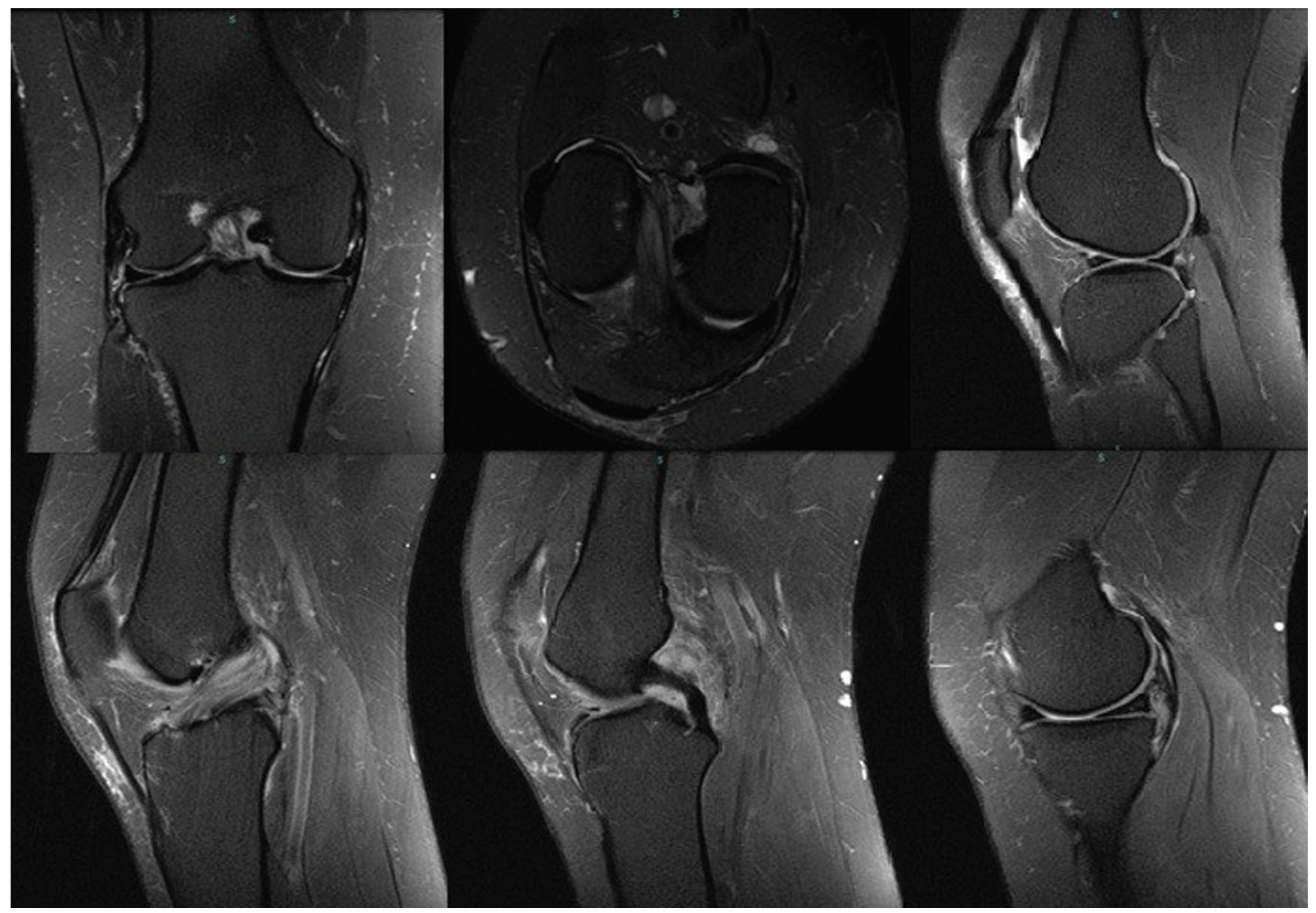

Fig. 3 Ressonância magnética ponderada em T2 demonstrando hipertrofia do ligamento cruzado anterior (LCA), e aumento de sinal sem perda da continuidade de suas fibras - sinal do "talo de aipo".

Optamos pelo tratamento cirúrgico videoartroscópico. Evidenciamos na artroscopia o LCA amarelo/acastanhado, hipertrofiado, e com tensão preservada de suas fibras (-Figura 4A e 4B). Realizamos a ressecção da porção degenerada e o desbridamento parcial das fibras comprometidas do LCA ( - Figura 4C e 4D). Não evidenciamos outras lesões associadas. $\mathrm{O}$ estudo histológico confirmou a hipótese diagnóstica, evidenciando a presença de substância mucoide difusa entremeada às fibras de colágeno. Após quatro sema-

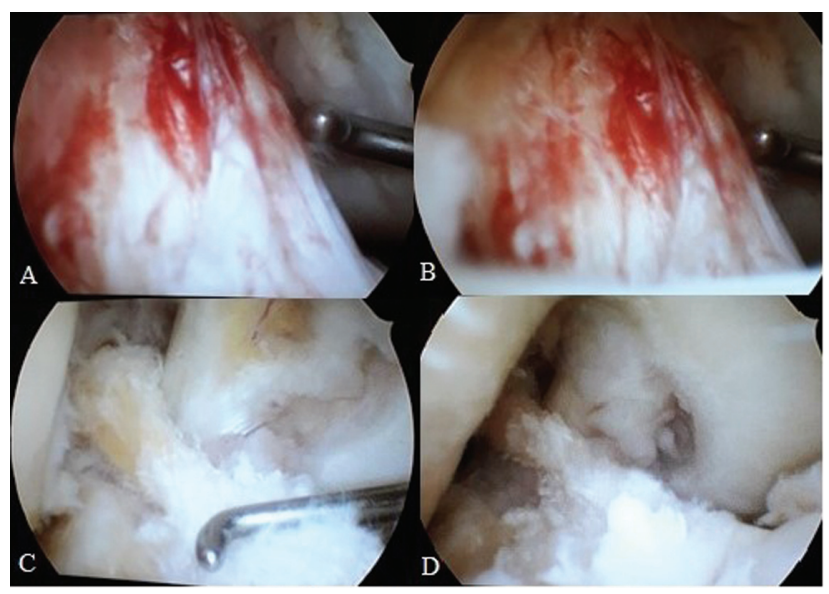

Fig. 4 Imagem peroperatória de artroscopia de joelho direito (A e B) evidenciando sinais de degeneração mucoide do LCA; (C e D) aspecto após desbridamento e ressecção parcial da porção degenerada do LCA. nas de pós-operatório, a paciente já apresentava alívio sintomático. Após 12 e 24 semanas de pós-operatório, repetimos a avaliação clínica, e evidenciamos objetivamente a melhora sintomática referida pela paciente sem o desenvolvimento de instabilidade (- Figura 5).

\section{Discussão}

A degeneração mucoide do LCA ainda é uma patologia pouco conhecida. Geralmente acomete homens entre 40 e 50 anos de idade, e frequentemente é confundida com ruptura parcial do LCA. ${ }^{2-6}$ É determinada por depósitos intersticiais de glicosaminoglicanos em meio aos feixes de colágeno, causando hipertrofia do LCA, dor no joelho, e limitação do arco de movimento, geralmente limitando os extremos da extensão ou flexão. ${ }^{7}$

A dor posterior que acomete a fossa poplítea é o achado mais consistente. ${ }^{2,4,7}$ É atribuída ao efeito de massa do LCA na chanfradura e/ou ao encarceramento patológico do LCA no compartimento femoro-tibial posterior, e está presente em $80 \%$ dos casos seguida pela limitação da flexão, que afeta em torno de $50 \%$ dos pacientes, que não respondem completamente aos AINEs e à fisioterapia. $3,8,9$

Kumar et al. $^{3}$ foram os primeiros a descrever o caso de um paciente de 35 anos de idade que apresentava dor na face posterior do joelho associada a diminuição do arco de movimento.

Múltiplas teorias tentam explicar a patofisiologia desta lesão. A teoria sinovial sugere que haja um acúmulo de 


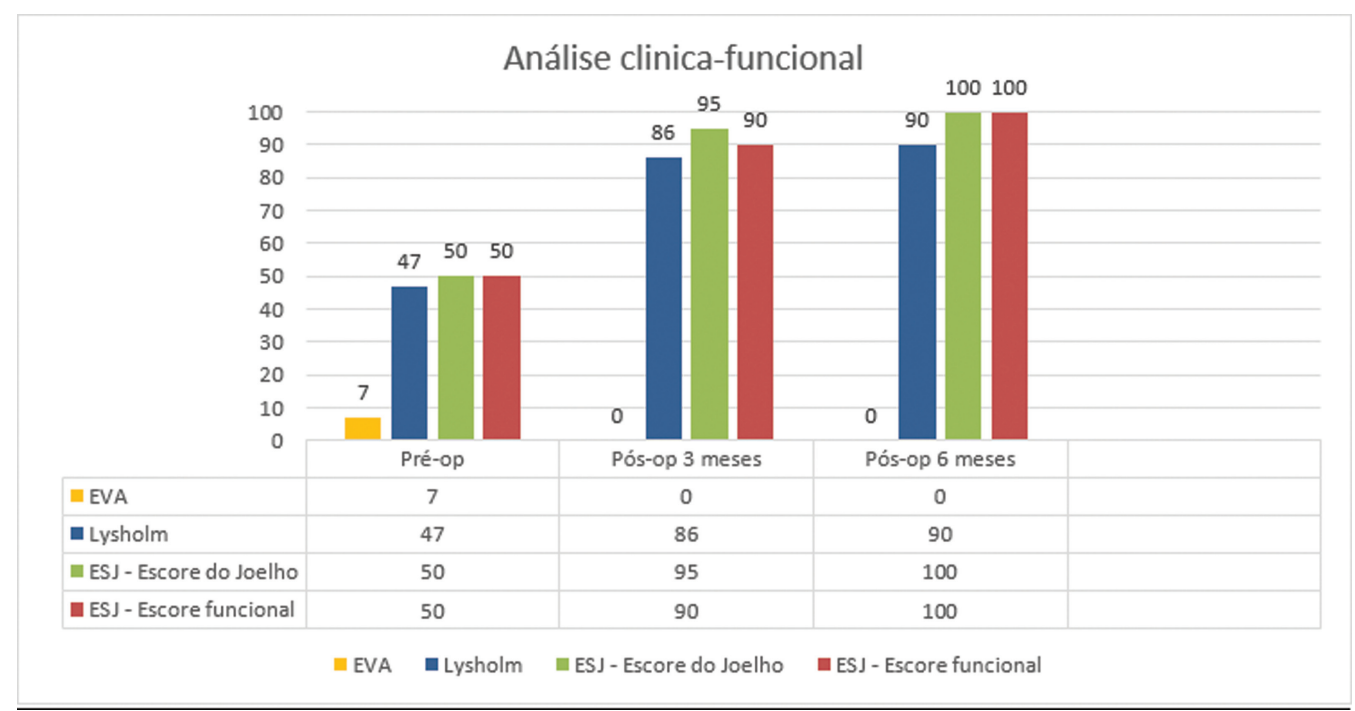

Fig. 5 Gráfico 1-análise clínico-funcional.

líquido sinovial por meio da herniação da membrana sinovial do LCA. A teoria traumática baseia-se na evidência da secreção de glicosaminoglicanos pelos fibroblastos do LCA em resposta a lesões traumáticas e a teoria degenerativa relaciona a presença de tecido mucoide como parte do envelhecimento normal deste tecido., ${ }^{4,9}$

O exame padrão ouro para o diagnóstico é a RM. A prevalência na RM varia de $1,8 \%$ a 5,3\%. ${ }^{2,4}$ McIntyre et al. ${ }^{5}$ demonstraram que os sinais de degeneração mucoide do LCA podem ser confundidos com a ruptura deste ligamento ao exame de RM, reforçando a importância da correlação clínico-radiológica para o diagnóstico correto.

Fernandes et al. ${ }^{1}$ relataram que, nessa patologia, o LCA se apresenta espessado, com fibras intactas entremeadas por material com intensidade de sinais de intermediária a baixa em imagens ponderadas em T1, e de sinal intermediário a alto em sequências ponderadas em T2 (sinal de "talo de aipo"), na RM que podem ser confundidas com rupturas parciais do LCA.

O diagnóstico deve ser confirmado histologicamente por exame patológico, principalmente nos casos associados a erosões ósseas. As lesões mucoides contêm uma substância basofílica marrom ou amarela, descrita como espessa ou densa, que contém alta densidade de glicosaminoglicanos. ${ }^{2,9}$

A artroscopia é utilizada tanto para o diagnóstico quanto para o tratamento definitivo. ${ }^{2,6}$ O LCA apresenta-se caracteristicamente aumentado em seu volume de cor amarela ou marrom-acastanhada, por vezes sem o revestimento sinovial. As fibras do LCA são contínuas e com orientação usual. A tensão geralmente é normal ou até mesmo aumentada. 0 pinçamento no intercôndilo pode ser evidenciado durante a avaliação artroscópica, o que pode explicar o déficit de extensão visto em alguns pacientes, bem como os sintomas tipicamente encontrados em casos de lesões meniscais, levando ao diagnóstico equivocado., 4

Um dos receios com o desbridamento do LCA é o desenvolvimento de instabilidade pós-operatória. Kumar et al. $^{3}$ consideraram a ressecção total do LCA como opção de tratamento que não resulta em queixas de instabilidade pós-operatória. Entretanto, a maioria dos autores $2,4,5,7$ concordam que a ressecção do tecido mucoide com debridamento parcial do LCA é suficiente. Dejour et al. ${ }^{10}$ relataram, em sua análise de 27 pacientes, a presença de testes de gaveta anterior positivos em 36\% e de deslocamento do pivô em $55 \%$ após debridamento parcial. Lintz et al. $^{2}$ demonstraram $93 \%$ de lassidão anterior após a ressecção da degeneração mucoide; entretanto apenas 2 de 29 pacientes necessitaram de reconstrução do LCA posteriormente.

No caso relatado neste artigo, também não evidenciamos instabilidade após a ressecção parcial do LCA em um seguimento de seis meses. Não há evidências na literatura de recidivas após a ressecção.

A degeneração mucoide do LCA deve ser lembrada na investigação dos casos sugestivos de ruptura parcial associada a dor posterior no joelho. A realização da RM é elucidativa. $\mathrm{O}$ estudo anatomopatológico define o diagnóstico. $\mathrm{O}$ tratamento envolve a ressecção do material mucoide, com desbridamento parcial do LCA o mais conservador possível, com a maioria dos autores ${ }^{3,7,9}$ relatando bons resultados sem ocorrência de instabilidade pós-operatória.

\section{Conflito de Interesses}

Os autores declaram não haver conflito de interesses.

\section{Referências}

1 Fernandes JL, Viana SL, Mendonça JL, et al. Mucoid degeneration of the anterior cruciate ligament: magnetic resonance imaging findings of an underdiagnosed entity. Acta Radiol 2008;49(01): 75-79

2 Lintz F, Pujol N, Boisrenoult P, Bargoin K, Beaufils P, Dejour D. Anterior cruciate ligament mucoid degeneration: a review of the literature and management guidelines. Knee Surg Sports Traumatol Arthrosc 2011;19(08):1326-1333 
3 Kumar A, Bickerstaff DR, Grimwood JS, Suvarna SK. Mucoid cystic degeneration of the cruciate ligament. J Bone Joint Surg Br 1999; 81(02):304-305

4 Bergin D, Morrison WB, Carrino JA, Nallamshetty SN, Bartolozzi AR. Anterior cruciate ligament ganglia and mucoid degeneration: coexistence and clinical correlation. AJR Am J Roentgenol 2004; 182(05):1283-1287

5 McIntyre J, Moelleken S, Tirman P. Mucoid degeneration of the anterior cruciate ligament mistaken for ligamentous tears. Skeletal Radiol 2001;30(06):312-315

6 Narvekar A, Gajjar S. Mucoid degeneration of the anterior cruciate ligament. Arthroscopy 2004;20(02):141-146
7 Fealy S, Kenter K, Dines JS, Warren RF. Mucoid degeneration of the anterior cruciate ligament. Arthroscopy 2001;17(09):E37

8 Hensen JJ, Coerkamp EG, Bloem JL, De Schepper AM. Mucoid degeneration of the anterior cruciate ligament. JBR-BTR 2007;90 (03):192-193

9 Melloni P, Valls R, Yuguero M, Sáez A. Mucoid degeneration of the anterior cruciate ligament with erosion of the lateral femoral condyle. Skeletal Radiol 2004;33(06):359-362

10 Dejour D, Cohn J, Tavernier T. Anterior Cruciate Ligament Cyst or Spontaneous Mucoide degeneration? Clinical, radiological and histological outcomes. Rev Chir Orthop Repar Appar Mot 2005;91 (S8):67 